\title{
Voxel-based morphometry in Alzheimer's patients
}

\author{
Leslie C. Baxter ${ }^{\mathrm{a}}$ and Marwan N. Sabbagh ${ }^{\mathrm{b}}$ \\ ${ }^{a}$ Barrow Neurological Institute, St. Joseph's Hospital - CHW, Phoenix, AZ, USA \\ ${ }^{\mathrm{b}}$ The Cleo Roberts Center of Clinical Research, Sun Health Research Institute, Sun City, AZ, USA
}

We thank the editorial staff and Dr. Ghosh-Dastidar and colleagues for their commentary on our manuscript "Relationship of Cognitive Measures and Gray and White Matter In Alzheimer's Disease" [1]. Dr. GhoshDastidar and colleagues have provided an overview of voxel-based morphometry methodology as well as the strengths and limitations of utilizing this technique in investigating MRI features done during clinical studies of various diseases.

We agree that the sample size of this study is not sufficient to make general statements about its utility in investigating MRI changes in Alzheimer's disease. Instead, we hope the findings of this study will provide interest in larger studies that further characterize the underlying neural changes associated with cognitive measures. We hope that we provide an example of how voxel-based morphometry can help answer these basic questions about the commonly used cognitive tests. Another important point that is discussed by GhoshDastidar et al. is the use of a custom template for image registration. It may be true that study-specific custom templates limit our ability to directly compare to other studies, but this technique minimizes mis-registration due to the obvious differences in elderly compared to the MNI composite and other templates. These limitations will likely be resolved with studies such as the Alzheimer's Disease Neuroimaging Initiative, that will allow the creation of templates from studies of large numbers of elderly subjects. We look forward to seeing more studies of Alzheimer's disease using VBM as we believe this technology may have an important role in elucidating how the brain adapts to stimuli and disease.

\section{Reference}

[1] L.C. Baxter, D.L. Sparks, S.C. Johnson, B. Lenoski, J.E. Lopez, D.J.Connor and M.N. Sabbagh, Journal of Alzheimer's Disease 9 (2006), 253-260. 